\title{
Cognitive components and the ability to ignore and adapt to irrelevant stimuli: a key factor in open plan offices?
}

\author{
Fostervold, Knut Inge ${ }^{*}$ \\ Department of Psychology, University of Oslo, P.O.Box 1094, Blindern, Norway
}

\begin{abstract}
Employees differ in their apperception of open plan offices. Despite this, individual differences have been sparsely treated in the literature discussing effects of office design. The aim of the present study was to investigate how stimulus screening, working memory, and attention interrelate and affect important factors in the work environment. The study utilized a cross sectional design. Participants $(\mathrm{N}=35)$ were recruited among employees in four organizations. Working memory was assessed bye means of the Operation -Word Span Task (OSPAN) while attention was measured by the $\mathrm{d} 2$ test of attention. Dependent variables were job stress, job involvement, organizational commitment, and need for recovery. The results showed a main affect of stimulus screening on need for recovery and one subscale of organizational commitment. A main effect was also found for fluctuation rate across trials, a sub measure of attention, on need for recovery. The results revealed no indication of any mediator or moderator effects of either working memory or attention, on stimulus screening, for any of the dependent variables. Thus, the effect of stimulus screening seems not to be explained through individual differences in cognitive processes.
\end{abstract}

Keywords: Open plan office, individual differences, stimulus screening, memory, attention.

\section{Introduction}

Due to increased globalization and increased need for flexibility in the modern work life there has been a renewed interest in office design. A solution that has engendered much debate is the use of open plan offices. Their main advantage is increased flexibility and a reduction of the space needed. Advocates claim that the open plan offices also reduce hierarchies in the organization, increase communication and learning, improve work flow, and productivity $[15,1]$. Others argue that the open plan office is primarily haunted by increased noise, lack of privacy, and reduced productivity $[19,17]$.

Obviously, people differ in their view of the open plan office. Sometimes this difference could be ascribed to habitual thinking and skepticism towards changes in general, but in most cases the reasons are probably caused by genuine differences in the way we perceive and encounter our physical world. Although the literature is sparse, the concept of "Stimu- lus screening" has engendered some interest over the years. The concept, originally developed by Merhabian [13], describes the ability to ignore and adapt to irrelevant stimuli. According to Merhabian [13], this ability is unconscious and automatic and can not be trained. People that easily adapt and ignore irrelevant stimuli are labeled good screeners. The concept of stimulus screening has been used, although in a revised form, in studies investigating the effect of open plan offices [16]. The results indicate that good screeners report higher job satisfaction and job performance compared to poor screeners which seem to benefit the most from increased privacy in the work environment.

However, the measurement of stimulus screening is based on self reports. It is, thus difficult to know the basis for the ability. One possibility is that stimulus screening reflects differences in cognitive abilities linked to selective attention. To investigate this hypothesis, Maher and von Hippel [12] tested 109 participants by means of the Stroop test, a question-

${ }^{*}$ Corresponding author. E-mail: k.i.fostervold@psykologi.uio.no 
naire containing stimulus screening, and a range of other job related measures. The results indicate that although selective attention was related to employee satisfaction, no association was found between selective attention and stimulus screening. It is tempting to conclude that stimulus screening is not a cognitive ability. However, the usefulness of the Stroop test as a measure of general cognitive processes has been criticized for not measuring memory [8].

It is possible that a link between stimulus screening and cognitive abilities is present if memory functions are taken into account. This hypothesis was tested by means of two cognitive tests measuring working memory and selective attention.

\section{Methods}

\subsection{Design and procedures}

The study utilized a cross sectional design. Individual data were gathered by means of a subjective questionnaire and two cognitive tests. Four different white collar organizations in the Oslo region in Norway agreed to participate in the study. Open plan offices were the normal working area for all employees in the organizations.

An e-mail containing written information about the study was distributed to all employees in the organizations. The e-mail was sent from the management of the organization stating the purpose of the study, the procedures, and that participation in the study was voluntary. Shortly after the mailing containing the general information, all employees received an email with an invitation to participate in the study, and a link to an electronic questionnaire located at a secure website. Completed questionnaires were saved anonymously. In order to be able to pair individual results from the questionnaire and the cognitive tests, the participants were instructed to fill in, and remember, a four digit code of their own choice. A reminder was sent to the employees two weeks after the first invitation.

Employees who had completed the questionnaire were invited to complete the cognitive tests. The test administration was conducted individually, in a separate room, at each workplace, to ensure comparable test conditions. The participants were informed about the purpose of each test and their right to terminate participation at any point.

\subsection{Participants}

35 participants completed both the questionnaire and the cognitive tests. All participants were working in open plan offices. The sample consisted of 20 males and 15 females. The mean age was 34.4 years (SD. 6.6 years). The distribution of participants among the four organizations was 9, 7, 8, and 11 . Mean tenure was 2.9 years and $94.2 \%$ of the sample had education beyond high school. The results from the $\mathrm{d} 2$ test of attention were excluded for one participant due to technical problems.

\subsection{Cognitive measures}

Working memory Working memory span was measured by means of the Operation -Word Span Task (OSPAN). OSPAN is based on a dual task paradigm [25]. Tasks measuring individual memory span have shown reliable and valid measures of working memory [6].

OSPAN consists of simple arithmetic problems and lists of unrelated words. The arithmetic problems were presented one at a time, centered on a computer screen. Immediately after the math problem a word was presented on the computer screen. See figure 1, for an example. The math problem and word presentations are referred to as a "math/word presentation."

$$
(3 \times 4)-1=11 ? \text { Rød }(\text { Red })
$$

Fig 1. Example of a math/word presentation. (English translation in parenthesis)

The subjects were instructed to read the math problem and to indicate whether the given answer was correct or not, by pressing down the $\mathrm{R}$ key (right) or G key (wrong) on the keyboard. Reaction time was not recorded. The participants were told that the math problems were an important part of the test and that the number of correct responses was recorded.

Immediately after the response, a word was presented, which the participant read aloud. Then a new math/word presentation followed. The math/word presentations were organized in sequences containing from two to eight presentations. Each sequence defines a memory span. A total of $70 \mathrm{math} /$ word presentations were given, with each memory span tested twice in random order, two prevent the participants from predicting the sequence [9]. After each sequence (Memory span) a recall cue was presented 
and the subjects were told to recall aloud as many words as they could remember, in the order in which they appeared. Before the critical trials began, the subjects were given three practice trials.

Set-size score (all words in one a sequence remembered correctly) and total memory span (Correct words remembered despite wrong position) were recorded. The program running the test were programmed in E-prime and developed in house.

\subsubsection{Attention performance}

Attention performance was measured by means of the $\mathrm{d} 2$ test of attention [5]. The $\mathrm{d} 2$ test is a paper-andpencil test that requires the participant to detect three subtly different target stimuli among a set of distractors within a limited time frame. The test consists of 14 letter rows, each containing 47 letters. Each letter is supplied with one or more apostrophes, positioned either or both above and beneath the letter. The target is defined as the letter " $d$ " with two apostrophes. The participants are instructed to mark the targets as fast and with as few errors as possible. The participant is required to change line after 20 seconds. Speed and rule compliance are tested under a task-load condition. Performance on the $\mathrm{d} 2$ test reflects individual attention span, concentration ability and coordination.

Three measures were calculated based on the raw scores. Overall performance (TN-E) is calculated by subtracting omission and commission error count (i.e. the number of missing and false responses) from the total number of stimuli processed. Concentration performance $(\mathrm{CP})$ is calculated by subtracting commissions from the total count. Fluctuation rate across trials (FR) is calculated as the difference between the line possessing the most processed items and the line possessing the least. After a brief instruction, the participant was given an example and a line of practice before the test started. Total testing time was approximately 5 minutes.

\subsection{Subjective questionnaire}

In addition to measuring cognitive components the participants answered an electronic questionnaire. Apart from background questions and some other customized information, the questionnaire contained Norwegian versions of international scales.

\subsubsection{Stimulus Screening}

Individual differences in the ability to ignore and adapt to irrelevant stimuli were measured by a short- ened and modified version [16] of a scale original developed by Merhabian [13].

The measure consists of 10 items with each item rated on a 7-steps Likert scale. The items are summed into one single index. Cronbach's alpha in the present study was .82 .

\subsubsection{Job stress}

Subjective experience of job stress was measured by the Norwegian edition of the Job Stress Survey (JSS-N) [24,11]. The JSS-N comprises 30 statements rated twice by the respondents. The first time the respondent is asked to evaluate, on a 9-point scale, how stressful a situation is perceived compared to a given standard. The second time the respondent is asked to indicate, on a scale from 0 to $9+$ days, how often they have experienced the situation the last 6 months.

The JSS-N yields three main scales: a total stress index (JS-X), a severity scale (JS-S), and a frequency scale (JS-F). An example of the factor structure and use of JSS is found in Spielberger and Reheiser, [23].

\subsubsection{Job Involvement}

Job engagement was measured by the Job Involvement -role (JIR) scale [18]. JIR consists of 13 items aiming at how immersed employees experience themselves to be in their current work tasks. Each item was rated on a 7-steps Likert scale. The items are summed into one index, which showed a Cronbach's alpha of .75 .

\subsubsection{Organizational commitment}

Organizational Commitment (OC) was measured by means of a questionnaire developed by Slocombe and Dougherty [20]. The questionnaire contains 14 items with each item rated on a 7-steps Likert scale. Three indices are constructed from the questionnaire: belief in and acceptance of organizational goals, willingness to exert effort on behalf of the organization, and desire to remain a member of the organization. Cronbach's alpha was .65, .72 and .81 for the first, second and third index, respectively.

\subsubsection{Need for Recovery}

Need for recovery after work was measured by The Need for Recovery Scale [26,22]. The scale consists of 11 items measuring daily fatigue and recuperation from work with Yes/No response alternatives. The items are summated to yield one single 
index (alpha .71). The Need for Recovery scale has shown to predict long term health effects [22,21].

\subsection{Statistics}

Ordinary least squares regression analyses were used to examine the relationship between expected predictors (stimulus screening, operation span and attention performance), and the dependent variables (job stress, job involvement, organizational commitment, and need for recovery). Possible moderator effects were examined by adding interaction terms to the regression models, while potential mediator effects were examined following the steps of Baron and Kenny [2]. Predictors and possible moderator variables were centered prior to the regression analyses.

Inspection of the variables revealed that the two OSPAN variables (set size score and total memory score), the three $\mathrm{d} 2$ variables (TN-E, CP, and FR), and the variable need for recovery were not strictly normally distributed. To improve the normal distribution the variables were transformed by the natural logarithm. All analyses were performed with PASW statistics version 18 .

\section{Results}

\subsection{Preliminary analyses}

Table 1 shows the mean and standard deviation for the predictor variables and the dependent variables. Intercorrelations between the predictor variables, the dependent variables, and background variables are shown in table 2.

With regard to the background variables, negative correlations were observed between age and measures of attention. Males seem to have somewhat higher scores on working memory and stimulus screening than females. The correlation matrix shows that stimulus screening ability was significantly correlated with all the three subscales measuring different facets of organizational commitment. Good screeners seem to experience higher organizational commitment. Stimulus screening was also significantly correlated with need for recovery indicating that good screeners experience lower need for recovery.
Table 1

Mean and standard deviation of predictor and dependent variables.

\begin{tabular}{lcc}
\hline & Mean & SD \\
\hline Stimulus Screening & 43.94 & 9.58 \\
Ospan Set-size score & 28.51 & 13.76 \\
Ospan total memory span & 173.66 & 35.31 \\
d2 Overall performance (TN-E) & 420.35 & 61.26 \\
d2 Concentration perform. (CP) & 153.74 & 31.29 \\
d2 Fluctuation rate (FR) & 13.85 & 3.95 \\
Job Stress severity (JS-S) & 5.07 & 0.82 \\
Job Stress frequency (JS-F) & 3.75 & 1.44 \\
Job Stress total (JS-X) & 18.49 & 7.40 \\
Job Involvement & 66.97 & 9.94 \\
Belief in organizational goals & 34.31 & 5.18 \\
Desire to remain in the organization & 22.97 & 3.49 \\
Willingness to exert effort & 23.77 & 2.77 \\
Need for Recovery & 3.51 & 2.51 \\
\hline
\end{tabular}

Although the correlations between stimulus screening, working memory, and attention were not significant, moderate correlations were observed between stimulus screening and both measures of working memory ( $r=.33)$, and between stimulus screening and $\mathrm{d} 2$ fluctuation rate $(r=.26)$. A moderate correlation was also observed between total OSPAN memory span and $\mathrm{d} 2$ overall attention performance (TN-E) $(r=.29)$. Neither working memory nor attention was significantly correlated with any of the dependent variables.

\subsection{Regression analyses}

Due to the high intercorrelation found between the two measures of working memory (OSPAN set size and OSPAN total memory) separate analyses were performed for each measure to avoid any danger of multicolinearity. The same justification and procedure was also applied for the measures of attention TN-E and CP. 
Table 2

Intercorrelations between measures

\begin{tabular}{|c|c|c|c|c|c|c|c|c|c|c|c|c|c|c|c|c|c|}
\hline & 1 & 2 & 3 & 4 & 5 & 6 & 7 & 8 & 9 & 10 & 11 & 12 & 13 & 14 & 15 & 16 & 17 \\
\hline Stim. Screening & - & & & & & & & & & & & & & & & & \\
\hline Ospan Set-size score & .33 & - & & & & & & & & & & & & & & & \\
\hline Ospan total mem. span & .33 & $.91^{* *}$ & - & & & & & & & & & & & & & & \\
\hline d2 Overall perf.(TN-E) & .03 & .20 & .29 & - & & & & & & & & & & & & & \\
\hline d2 Concen. perf. (CP) & -.10 & .30 & .25 & $.72^{* *}$ & - & & & & & & & & & & & & \\
\hline d2 Fluctuat. rate $(F R)$ & .26 & .07 & .22 & .15 & -.19 & - & & & & & & & & & & & \\
\hline Stress severity (JS-S) & -.14 & -.08 & -.04 & .14 & .25 & .05 & - & & & & & & & & & & \\
\hline Stress frequency (JS-F) & .10 & .09 & -.02 & .01 & .08 & -.03 & -.09 & - & & & & & & & & & \\
\hline Job Stress tot. (JS-X) & -.02 & .08 & .01 & .07 & .18 & -.01 & .25 & $.91^{* *}$ & - & & & & & & & & \\
\hline Job Involvement & .18 & .24 & .16 & -.02 & -.08 & .22 & -.03 & .07 & .02 & - & & & & & & & \\
\hline Belief in org. goals & $.49^{* *}$ & -.08 & -.02 & .06 & -.03 & -.07 & .08 & .10 & .03 & $.40^{*}$ & - & & & & & & \\
\hline Desire to remain & $.36^{*}$ & .10 & .18 & -.17 & -.22 & .11 & .24 & .14 & .17 & .29 & $.60^{* *}$ & - & & & & & \\
\hline Willing to exert effort & $.35^{*}$ & -.00 & -.06 & .09 & .10 & -.03 & .03 & .26 & .16 & $.43^{* *}$ & $.70^{* *}$ & $.61^{* *}$ & - & & & & \\
\hline Recovery & $-.38^{*}$ & -.00 & -.01 & .04 & -.12 & .25 & .02 & .32 & $.40^{*}$ & .05 & $-.40^{*}$ & -.10 & -.23 & - & & & \\
\hline Age & .14 & -.07 & -.09 & $-.37^{*}$ & -.22 & .04 & .14 & -.14 & -.00 & .05 & .07 & -.01 & -.01 & .10 & - & & \\
\hline Education & .02 & -.13 & -.20 & .30 & .18 & -.21 & .14 & .23 & .26 & -.10 & .03 & -.22 & -.16 & -.02 & -.19 & - & \\
\hline Tenure & -.09 & .14 & .11 & -.19 & -.08 & .05 & -.05 & .11 & .18 & .17 & -.01 & .00 & .08 & .22 & $.60^{* *}$ & -.31 & - \\
\hline Gender & $.40^{*}$ & $.36^{*}$ & .30 & .19 & -.00 & .17 & .23 & .07 & .15 & .07 & .07 & .03 & .03 & .10 & .21 & .18 & .25 \\
\hline
\end{tabular}

$* \mathrm{p} \leq .05, * * \mathrm{p} \leq .01$.

\subsection{Mediator analyses}

Meditation effects presuppose the presence of a correlation between the independent variable and the presumed moderator variable [2]. The observed zeroorder correlations were not significant for any of the cognitive measures. However, due to the size of the correlations, moderator analyses, with stimulus screening as predictor variable, were performed on both measures of working memory and $\mathrm{d} 2$ fluctuation rate. The analyses were performed both with Need for recovery and Belief in and expectance of organizational goals as dependent variable.

The results revealed no indication of any mediating effects of either measure of working memory or d2 fluctuation rate, on stimulus screening, for any of the dependent variables.

\subsection{Moderator analyses}

Age and gender were included in the analyses of possible moderator effects due to high intercorrelations with some of the predictor variables.

Main effects of stimulus screening were observed with regard to one of the subscales of organizational commitment (Belief in and acceptance of organization goals) and need for recovery. The results indicate that good screeners have lower need for recovery and that good screeners seem to have higher belief in and acceptance of organizational goals. A main effect of fluctuation rate across trials (FR) was observed for need for recovery. The result indicate that poor screeners have higher fluctuation rate compared to good screeners, or put differently, that they have larger difference between the line possessing the most processed items and the line possessing the least. Statistical details for the analyses, showing the 
highest and lowest effects, are shown in table 3 for all three dependent measures.

\section{Table 3}

Statistical details for main effects

\begin{tabular}{lcccc}
\hline \multicolumn{1}{c}{ Variables } & Std. Error & Beta & $\mathrm{t}$ & Sig. \\
\hline $\begin{array}{l}\text { Dependent varable: } \\
\text { Need for recovery }\end{array}$ & & & & \\
Stimulus screen, Lowest & .013 & -.551 & -2.849 & .008 \\
Stimulus screen, Highest & .012 & -.629 & -3.455 & .002 \\
Fluctuation rate, Lowest & .333 & .350 & 2.196 & .037 \\
Fluctuation rate, Highest & .330 & .363 & 2.301 & .029 \\
\hline $\begin{array}{l}\text { Dependent variable: } \\
\text { Belief in organizational goals }\end{array}$ & & & & \\
Stimulus screen, Lowest & .105 & .571 & 2.832 & .009 \\
Stimulus screen, Highest & .10 & .592 & 3.088 & .005 \\
\hline
\end{tabular}

The results revealed no significant interactions between the predictor variables for any of the dependent variables.

\section{Discussion}

The aim of the present study was to investigate how stimulus screening, working memory, and attention interrelate and affect important psychosocial factors in open plan offices.

The results did not reveal any mediator or moderator effects. The supposed link between stimulus screening and cognitive abilities, measured as working memory and selective attention, was not supported. This result is in line with the results reported by Maher and von Hippel [12]. Thus, the present finding seems to support the idea that stimulus screening is an independent concept that is not heavily influenced by cognitive factors.

Previous studies have shown that individual differences in stimulus screening ability affect a diversity of work related factors such as: job satisfaction, performance, stress, coping with crowding, and cognitive appraisals of the environment $[3,16,12,7]$.

The main effects of stimulus screening found in this study concur with this general trend, and adds additional evidence to the importance of stimulus screening as an important factor in the discussion of office design. The finding showing that good screeners experience less need of recovery from work com- pared to poorer screeners fits nicely to the theoretical model proposed for stimulus screening [13]. In this model stimulus screening is seen as the ability to ignore and adapt to irrelevant stimuli. According to the theory, screening ability is connected to arousal. People who are poor screeners experience higher arousal and stronger emotions, both negative and positive, when encountering high-information events, compared to people who are good screeners. It follows that poor screeners take longer time to get back to a normal unemotional state [14]. Being in an elevated state of emotional arousal for longer periods ostensibly takes its toll and may lead to higher need for recovery.

This explanation may also apply for the finding that good screeners tend to experience higher belief in organizational goals, a subscale of organizational commitment, than poor screeners. Being in an elevated emotional state more often, poor screeners may experience stronger emotional feelings towards the goals of the organization, both in a negative and positive sense. It is plausible to assume that high emotional variability may make more difficult to develop stable identification and acceptance of the goals of the organization.

The results also revealed a main effect of fluctuation rate across trials, were high fluctuation rate was associated with higher need for recovery. It seems difficult to provide a straight forward explanation for this finding. However, a possibility may be in the line of thinking behind theories of self-regulation and ego depletion [4]. According to this thinking, self regulation relies on a limited resource. When used, the person will no longer be able to continue the self regulating process and will fall into a state of ego depletion. In this state, efforts to retain self regulation will be less than normally successful. Self regulatory tasks are not necessarily particularly difficult or effortful. Given the material used in the $\mathrm{d} 2$ test of attention it is quite plausible to assume that the test require high amounts of self regulation. A possible explanation may therefore be that people displaying high fluctuation rate have lower self regulating capacity than people with low fluctuating rate. Being in a state of ego depletion they experience it more difficult to maintain self regulation at a steady state throughout the whole test. Although it is still under debate; ego depletion has been connected to fatigue, [10] and consequently also to need for recovery.

The result may also be regarded a cautious support for previous findings showing that cognitive factors, measured by the Stroop task, influence people's reactions to open plan offices. The hesitation hinges on 
the fact that although the $\mathrm{d} 2$ test of attention correlates with the Stroop Color Word test, only minor and insignificant correlations have been shown between the Stroop task and the sub measure of fluctuation rate (FR) in the $\mathrm{d} 2$ test [5].

In conclusion, the results obtained in this study points towards stimulus screening as an important factor in the understanding of individual differences in people's appraisal of the office environment. Some support is also present for the importance of cognitive factors in this regard. Further, the effect of stimulus screening seems not to be explained through individual differences in cognitive processes.

\section{Acknowledgements}

The author wants to acknowledge the assistance with ideas and data collection by Maria Baastad, Kristin Opsahl, and Åsmund Lind.

The author would also express his appreciation for the kind co-operation of the employees and management of the organizations where the study was performed.

\section{References}

[1] R. Appel-Meulenbroek, Knowledge sharing through copresence: added value of facilities, Facilities 28 (2010), 189 205.

[2] R.M. Baron, D.A. Kenny, The moderator-mediator variable distinctionin social psycho-logical research: conceptual, strategic, and statistical consid-erations, Journal of personality and social psychology 51 (1986), 1173-1182.

[3] A. Baum, L.E. Calesnick, G.E. Davis, R.J. Gatchel, Individual differences in coping with crowding: Stimulus screening and social overload., Journal of Personality and Social Psychology 43 (1982), 821-830

[4] R.F. Baumeister, M. Gailliot, C.N. DeWall, M. Oaten, SelfRegulation and personality: how Interventions increase regulatory success, and how depletion moderates the effects of traits on behavior, Journal of personality 74 (2006), 1773 1801 .

[5] R. Brickenkamp, E. Zillmer, d2 test of attention, Hogrefe \& Huber Publish, Seattle Toronto Bern Göttingen, 1998.

[6] A.R.A. Conway, M.J. Kane, M.F. Bunting, D.Z. Hambrick, O. Wilhelm, R.W. Engle, Working memory span tasks: A methodological review and user's guide., Psychonomic Bulletin \& Review 12 (2005), 769-786.

[7] K. Dijkstra, M.E. Pieterse, A.T.H. Pruyn, Individual differences in reactions towards color in simulated healthcare environments: The role of stimulus screening ability, Journal of Environmental Psychology 28 (2008), 268-277.

[8] E. Elliott, K.M. Barrilleaux, N. Cowan, Individ-ual differences in the ability to avoid distracting sounds, European Journal of Cognitive Psychology 18 (2006), 90-108.

[9] W.R. Engle, J. Cantor, J. Carullo, Individual differences in working memory and comprehension: A test of four hypotheses. , Journal of Experimental Psychology: Learning, Memory, and Cognition 18 (1992), 972-992.

[10] S. Gross, N.K. Semmer, L.L. Meier, W. Kälin, N. Jacobshagen, F. Tschan, The effect of positive events at work on after-work fatigue: They matter most in face of adversity, Journal of Applied Psychology 96 (2011), 654-664.

[11]K. Håseth, Norwegian adaptation of the Job Stress Survey., in: C.D. Spielberger, I. Sarason (Eds.), Stress and Emotion: anxiety, anger, and curiosity, Taylor \& Francis, Washington DC, 2005, pp. 293-311.

[12]A. Maher, C. von Hippel, Individual differences in employee reactions to open-plan offices, Journal of Environmental Psychology 25 (2005), 219-229.

[13]A. Mehrabian, A questionnaire measure of individual differences in stimulus screening and associated differences in arousability, Journal of Nonverbal Behavior 1 (1977), 89-103.

[14]A. Mehrabian, Theory and evidence bearing on a scale of Trait Arousability, Current Psychology 14 (1995), 3-28.

[15]G. Mylonas, J.R. Carstairs, Open Plan Office Environments: Rhetoric and Reality, in: A. Glendon, B.M. Thompson, B. Myors (Eds.), Advances in Organisational Psychology, Australian Academic Press, Bowen Hills, Qld., 2008, pp. 443455.

[16]G.R. Oldham, C.T. Kulik, L.P. Stepina, Physical environments and employee reactions: Effects of stimulusscreening skills and job complexity, The Academy of Management Journal 34 (1991), 929-938.

[17] V.G. Oommen, M. Knowles, I. Zhao, Should health service managers embrace open plan work environments? A review, Asia Pacific Journal of Health Management 2 (2008) 37-43.

[18]I.M. Paullay, G.M. Alliger, E.F. Stone-Romero, Construct validation of two instruments designed to measure job involvement and work centrality, Journal of Applied Psychology 79 (1994), 224-228.

[19] J. Pejtersen, L. Allermann, T.S. Kristensen, O.M. Poulsen, Indoor climate, psychosocial work environment and symptoms in open-plan offices, Indoor Air 16 (2006), 392401.

[20] T.E. Slocombe, T.W. Dougherty, Dissecting organizational commitment and its relationship with employee behavior, Journal of business and psychology 12 (1998), 469-491.

[21]J.K. Sluiter, A.J. van der Beek, M.H.W. Frings-Dresen, The influence of work characteristics on the need for recovery and experienced health: a study of coach drivers, Ergonomics in design 42 (1999), 573-583.

[22]J.K. Sluiter, E.M. de Croon, T.F. Meijman, M.H.W. FringsDresen, Need for recovery from work related fatigue and its role in the development and prediction of subjective health complaints, Occupational \& Environmental Medicine 60 (2003), i62-i70

[23] C.D. Spielberger, E.C. Reheiser, Job stress in university, corporate, and military personnel, International Journal of Stress Management 1 (1994), 19-31.

[24]C.D. Spielberger, P.R. Vagg, Job Stress Survey - Professional manual, Psychological Assessment Resources. Inc, Odessa. FL, 1999.

[25] M.L. Turner, R.W. Engle, Is working memory capacity task dependent?, Journal of Memory and Language 28 (1989), 127-154.

[26]M. van Veldhoven, S. Broersen, Measurement quality and validity of the "need for recovery scale", Occupational \& Environmental Medicine 60 (2003), i3-i9. 